\title{
Posibilidad y utilidad de la Teoría Positiva de la Contabilidad
}

\section{R.D. WHITLEY}

Escuela de Negocios

de la Universidad de Manchester
Artículo publicado originalmente en inglés: "The Possibility and Utility of Possitive Accounting Theory", Accounting, Organizations and Society, Vol 13 No. 6, pp. 31-645, 1988. Traducción: Antonio Navarrete. Revisión: Bernardo Barona Z.
Resumen

$\int \begin{aligned} & \text { a Teoría Positiva de la } \\ & \text { Contabilidad de Watts y } \\ & \text { Zimmerman (Englewoods Cliffs, }\end{aligned}$ NJ: Prentice-Hall, 1986) festeja el crecimiento de la investigación "científica" de la contabilidad en los Estados Unidos y el progreso intelectual que ha realizado. Sin embargo, la extensión con que este tipo de investigación sigue los preceptos de las filosofias empiristas de la ciencia, es materia de algún debate. Más aún, la coherencia y aplicabilidad de las reglas metodológicas que Watts y Zimmerman aseguran seguir, es cuestionable. En particular, el programa Popperiano de investigación es de dudosa utilidad para los investigadores de la contabilidad. Las características especiales de los fenómenos sociales también plantean dudas sobre la posibilidad de modelos de ingeniería para tecnologías sociales basadas en la teoría. Estas dudas sobre el valor práctico y epistemológico de la "teoría positiva de la contabilidad" conducen a una explicación alterna, sociológica, de su desarrollo e institucionalización en la investigación académica de la contabilidad. 
La publicación de la Teoría Positiva de la Contabilidad por Watts y Zimmerman (1986) provee una oportunidad para reflexionar sobre el considerable crecimiento en la investigación académica, de prácticas contables y su estilo dominante, especialmente en las culturas anglo-sajonas. Como lo han observado muchos autores, la investigación contable se ha convertido en una actividad central para muchos académicos de escuelas de negocios y ha cambiado substancialmente de dirección hacia una preocupación más formal y deductiva con prueba de hipótesis y métodos cuantitativos (Dyckman y Zeff, 1984; Hopwood y Brownwich, 1984; Kaplan, 1986; Mattessich, 1972). Es el nuevo estilo de investigación que celebran Watts y Zimmerman como "científico" y que ellos consideran satisface una nueva demanda de conocimiento. De muchas maneras se puede ver este libro como un manifiesto de tal investigación "cientifica" de la contabilidad y de su posición de liderazgo en el dominio de la contabilidad académica en los Estados Unidos. A pesar de la demolición que Christenson hizo de las pretensiones científicas de la escuela de "Rochester" sobre la investigación contable y de los ataques de otros sobre su explicación del crecimiento de la investigación académica (Lowe et al., 1983; cf. Peasnell y Williams, 1986), Watts y Zimmerman continúan presumiendo el estatus científico del nuevo estilo de la investigación contable y su utilidad práctica sin justificación sistemática.
Al considerar esta expansión de la investigación académica y su dominio por un estilo y sistema de creencias particulares, me concentraré en este ensayo sobre la suficiencia del modelo dominante de "ciencia" que muchos investigadores de la contabilidad asumen como una teoría de método científico y su propiedad para la investigación contable. Después de discutir brevemente el criterio de Watts y Zimmerman sobre la teoria positiva de la contabilidad y el alcance hasta el cual ésta sigue las reglas metodológicas que ellos aseguran ser constitutivas del saber científico, considero algunas de las dificultades asociadas con el programa Popperiano de investigación en la filosofía de la ciencia. Me centro en la teoría del método científico de Popper porque tanto Watts y Zimmerman como algunos de sus críticos, como Christenson, aseguran seguirla. Lüego, considero si las reglas metodológicas para evaluar pretensiones de conocimiento en las ciencias naturales pueden

generalizarse a las ciencias sociales, como Watts y Zimmerman y muchos otros asumen, y, las consecuencias de ciertos rasgos de fenómenos y procesos sociales en la producción y evaluación del conocimiento en las ciencias. Estos rasgos también afectan el uso de teorías contables para el mejoramiento de convenciones y prácticas contables. Finalmente, sugeriré brevemente algunas de las razones sociológicas de por qué la investigación de la contabilidad ha crecido tanto y ha sido dominada por enfoques y métodos cuantitativos formales. 


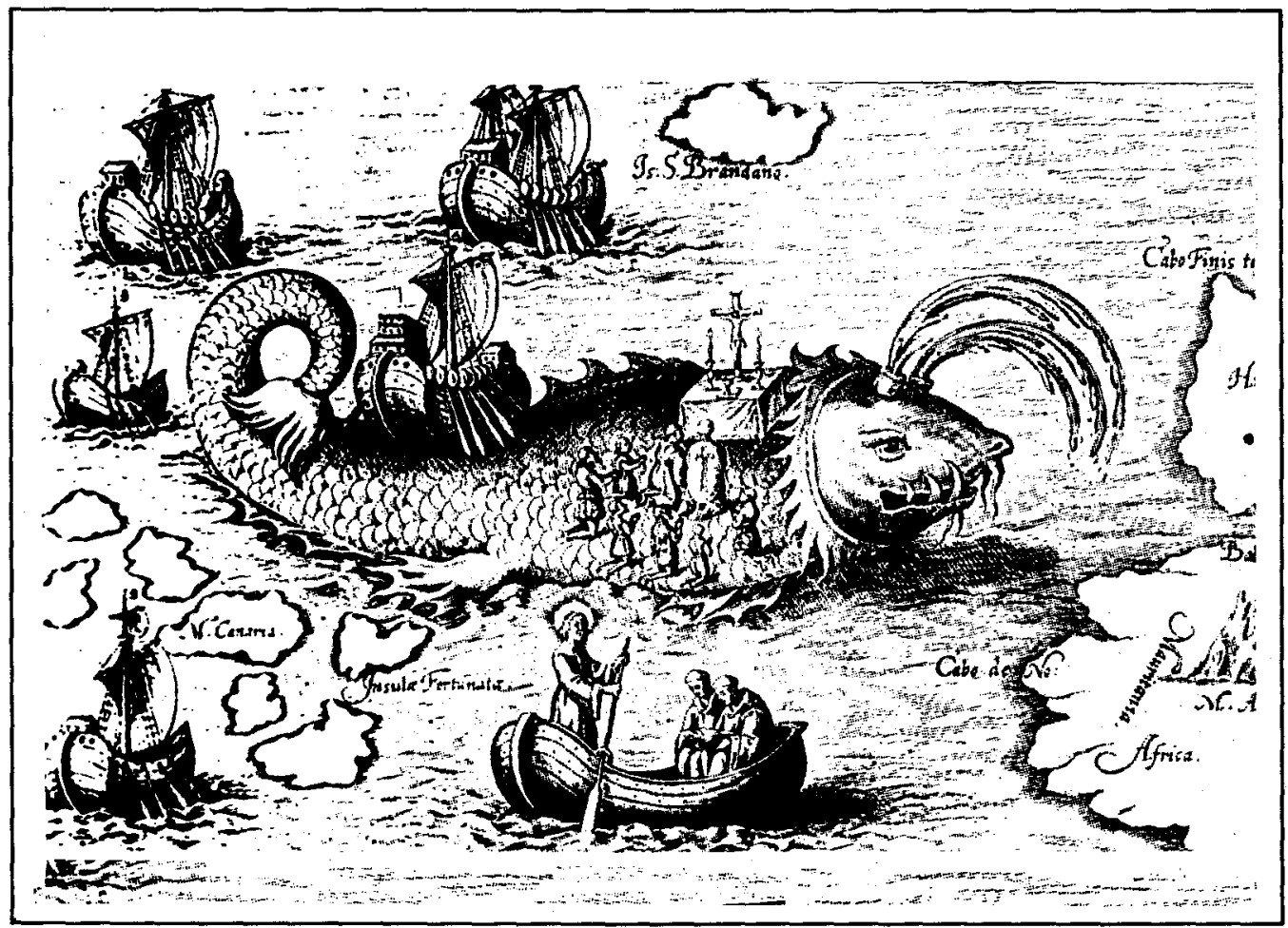

\section{Naturaleza y estatus de la Teoría Positiva de la Contabilidad}

egún Watts y Zimmerman (1986, p. 2), la teoría positiva de la contabilidad se preocupa de la explicación y predicción de prácticas contables. Da razones para las prácticas observadas y predice la ocurrencia de fenómenos contables inadvertidos.

Es "positiva" porque maneja hechos relacionados con la forma en que trabaja el mundo, en contraste con teorías y declaraciones normativas sobre cómo debería trabajar el mundo. Arguyendo la autoridad de Milton Friedman y otros economistas para esta distinción, los autores parecen olvidadizos de la considerable literatura crítica sobre economía "positiva" (ver, por ejemplo, Caldwell, 1982, capítulos 8-9; Coddington, 1972; Eichner, 1983; Musgrave, 1981) y las limitaciones de las filosofías empíricas de la ciencia. También parecen ignorar las recientes discusiones sobre la conveniencia de las metodologías científicas naturales para las ciencias humanas y los modelos de ingeniería para las tecnologías de intervención social (Bhaskar, 1979, capítulos 2-3; Fay, 1975, capítulos 2-3; Stockman, 1983, capítulos 6-7; Thomas, 1979, capítulos 3-4; Turner, 1980). La idea de que los investigadores contables pueden simplemente construir teorías generales de prácticas contables que les permitan a los profesionales predecir los resultados de sus decisiones en un mundo incierto, siguiendo reglas metodológicas basadas en la economía neo-clásica del equilibrio, es, por decir 
lo menos, tendenciosa y expuesta a considerable escepticismo. La habilidad de Watts y Zimmerman para ignorar mucha de la literatura en la filosofía de las ciencias naturales y sociales mientras hacen asertos metodológicos, ejemplifica la naturaleza fragmentada de la investigación contable como campo intelectual y la carencia de severas normas teóricas que integran los resultados de la investigación (Whitley, 1984).

Watts y Zimmerman consideran que la reciente investigación contable está constituida principalmente por "pruebas" empíricas de teorías basadas en la economía acerca del papel de la información contable en los cambios en el mercado. Aunque si se refieren a algún otro trabajo, la parte principal de su alegato de la teoría positiva de la contabilidad se centra en el alcance de las teorías del equilibrio para los mercados de capital y los efectos desequilibradores de la nueva información contable. Así, hay capítulos que resumen la investigación de la Hipótesis de los Mercados Eficientes y del Modelo de Valoración de Activos de Capital, los efectos de anuncios de ganancias, de revelación de regulaciones y de cambios en convenciones contables sobre el movimiento del precio de acciones, de contratación agencial y de la selección administrativa de las convenciones. Prácticamente toda esta investigación presume que los modelos de mercado de la economía neo-clásica y las pruebas de hipótesis por medio de análisis estadísticos de bases estándar de datos, derivados de los informes anuales y de información pública similar son adecuados. Así da a entender que sigue el modelo del trabajo científico popularizado por filósofos de la ciencia lógico-positivista y lógico-empirista en el que los científicos desarrollan teorías y leyes generales que luego son puestas a prueba contra pronunciamientos de observación basados en la experiencia. Según Watts y Zimmerman, este tipo de investigación ha mostrado progreso porque las teorías han sido reemplazadas por otras más refinadas y se han corregido los errores técnicos. De esta manera ellos resumen el desarrollo de la investigación empírica de la contabilidad como una serie de mejoras teóricas y refinamientos técnicos.

Dejando de lado la cuestión de si esta selección de investigación es completamente representativa de la reciente investigación contable, y si la investigación "positiva" es el único tipo digno de resumirse, parece dudoso que estos estudios sí sigan las reglas metodológicas promulgadas por filósofos como Hempel y Popper. Christenson (1983) ya ha puntualizado el fracaso de la escuela de Rochester en probar sus teorías sistemáticamente y los ajustes ad-hoc hechos por Watts y Zimmerman cuando encararon aparentes falsificaciones de sus hipótesis.

Similarmente, Lowe et al. (1983) produjeron evidencia empírica que parece contradecir su relato del desarrollo de teorías de la contabilidad en su artículo de 1979; sin embargo, la mayoría del trabajo es reproducido como explicación del crecimiento de la investigación contable en este libro. Este planteamiento caballeroso de las 
pruebas empíricas hace eco a su bastante sencilla exposición del cambio y desarrollo intelectuales que asume que el progreso científico consiste en mejoras metodológicas para dar cuenta de las anomalías. Mientras que Popper (1968, pp. 81-111) sugirió que los científicos deberían rechazar las teorías que fueran encontradas inconvenientes con las afirmaciones de observación, y el progreso consistía en reemplazar teorías falsificadas por aquellas con mayor contenido empírico y verosimilitud, Watts y Zimmerman parecen aplaudir la aceptación continuada del EMH y del CAPM a pesar de su inmunidad a las pruebas empíricas (Findlay y Williams, 1980) y también parecen incapaces de demostrar que el contenido empírico de las teorías de la contabilidad ha aumentado. Ciertamente no es claro cómo cualquier teoría que asegura describir los estados de equilibrio de mercados "perfectos", en la que la información es verdadera y gratis, pueda ser considerada con contenido empírico (cf. Whitley, 1986). Sin embargo, puesto que se han señalado puntos similares acerca de la economía, este tipo de investigación contable puede no ser peor que otras seudo-ciencias (Blaug, 1980, pp. 253-264; Caldwell, 1982; Deane, 1983; Hutchinson, 1976, 1984).

Si la investigación compendiada por Watts y Zimmerman no sigue los preceptos metodológicos que ellos aseguran caracterizan a la "ciencia", entonces el estatus epistemológico de sus pretensiones de conocimiento está claramente en duda. Por supuesto, una posibilidad adicional es que las reglas metodológicas de Popper son incoherentes y / o inapropiadas para saber de estas clases de fenómenos. En particular, pueden no ser aplicables a la investigación en las ciencias humanas (Findlay y Williams, 1985). Esta última posibilidad no es mencionada por Watts y Zimmerman porque ellos consideran que el método científico del deductivismo hipotético es aplicable a todos los fenómenos y que es el único camino válido de generar y tasar verdaderas pretensiones en todas las ciencias. La suficiencia general del informe de Popper sobre el progreso científico es igualmente ignorada por ellos que no dan razones de por qué preferir esta explicación, digamos, a las explicaciones realistas (Bhaskar, 1975; Harré) o muestran alguna indicación de que son conscientes de las alternativas.

Generalmente, Watts y Zimmerman parecen confiar mucho en la ortodoxia dominante de la economía neo-clásica al justificar la teoría positiva de la contabilidad y su libro podría verse como presentando un caso metodológico para reducir la investigación contable a una rama de la economía. Dependiendo del punto de vista que uno tenga sobre el estatus metodológico de la economía ortodoxa y su validez como empresa intelectual, esta fundación para la investigación contable puede parecer menos que segura, especialmente si los investigadores se preocupan de explicar por qué los eventos y fenómenos sociales ocurren y cambian y ayudan a resolver problemas prácticos: Como lo destacan Findlay y Williams $(1980,1985)$, sacar recomendaciones políticas de teorías que convierten la incertidumbre en riesgo, para 
analistas y administradores en un mundo incierto e internamente relacionado, es improbable que sea muy efectivo. Similarmente, presunciones de competencia "perfecta", expectativas homogéneas, información gratis y costos insignificantes en las transacciones alejan mucha de la economía financiera y la teoría positiva de la contabilidad del mundo de los profesionales y es incompatible con sus preocupaciones (Whitley, 1986). El dominio de tales presunciones en la economía, con perdón de Jensen (1972) y Ross (1978), no justifica automáticamente que sean correctas o demuestre su pertinencia para los problemas cotidianos en mundos sociales inciertos $\mathrm{e}$ interdependientes.

El estatus epistemológico de la reciente investigación sobre la contabilidad ha sido discutido por varios escritores en los últimos 5 años o algo más (e.g. Ryan, 1982; Thomas, 1989; Tomkins y Groves, 1983). En muchos casos, una posición filosófica particular ha sido asumida y luego usada para evaluar un cuerpo de investigación sin ponerle mucha atención al propósito y suficiencia de esa posición. Como hemos visto, Watts y Zimmerman reclaman la autoridad de Popper para su visión de la ciencia y su generalización para la investigación de la contabilidad, compromiso compartido por algunos de sus críticos, como Christenson (1983). Al considerar qué tan apropiado es tal compromiso para los investigadores contables y para los que exigen conjuntos particulares de reglas metodológicas, es evidentemente importante comprender el propósito de esas reglas y la extensión en que lo cumplen, así como su pertinencia para analizar el estatus epistemológico de la investigación contable.
Entonces, antes de discutir el punto de si las teorías del conocimiento científico natural son necesariamente aplicables a las ciencias humanas, brevemente expondré varios temas de discusión sobre la naturaleza del programa Popperiano de investigación en la filosofía de la ciencia y su éxito en proveer una teoría del progreso del conocimiento científico. Aunque me centro en el trabajo de Popper, muchos de los puntos en cuestión, son aplicables a otras formas del empirismo lógico.

\section{Propósito y suficiencia del programa Popperiano de investigación}

Una de las más importantes características de la teoría de Popper sobre el método científico es su intento de formular un conjunto de reglas metodológicas por medio de las cuales podría decirse que el conocimiento científico progresa. Esencialmente, Popper está preocupado por desarrollar una teoría "racional" del cambio científico que provea las reglas del juego para la ciencia como una empresa intelectual progresiva (Popper, 1968, pp. 53, 119-126, 276281; 1983, capítulo 10). Esta meta es diferente del intento lógico-positivista para formalizarel conocimiento científico existente como un verdadero sistema coherente y así establecer los criterios epistemológicos para valorar pretensiones de conocimiento sobre la base de la física aceptada actualmente. Popper rechazó tal "naturalismo científico" a causa de la falibilidad del conocimiento científico y especialmente de las observaciones empíricas (Popper, 1968, pp. 50-53). Echando por tierra la validez del conoci- 
miento empírico en la irrefutable experiencia del mundo natural, los positivistas presumían que la élite científica actual ha producido verdadero conocimiento que no puede ser revisado o radicalmente mejorado. Puesto que Popper pensó que la tarea básica de una teoría de la ciencia era dar cuenta del progreso a través del cambio intelectual, rechazó esta noción estática del conocimiento basada en la falaz doctrina de la inducción. En su opinión, la experiencia es siempre revisable y combatible y por consiguiente no puede proveer una base epistemológica firme e irrefutable para tasar las presunciones de conocimiento. Es, más bien, cómo la experiencia se usa para separar el conocimiento científico de otras formas de comprensión. Como lo sugiere él mismo (1968, p. 108): "La comprobación de una teoría depende de afirmaciones básicas cuya aceptación o rechazo, a su turno, depende de nuestras decisiones. Así, son las decisiones las que fijan el destino de la teorías".

Este convencionalismo metodológico sostiene que las convenciones que indican cómo los juicios y preferencias científicas son hechos, es lo que determina su validez y si conducen al progreso. Entonces, como una teoría del método científico, el planteamiento de Popper tiene que ser juzgado en términos de su coherencia para establecer reglas de decisión que den cuenta del avance científico y la probabilidad de que estas reglas puedan ser institucionalizadas en los sistemas de investigación. Puesto que este planteamiento se centra en cómo se toman las decisiones, o deberían tomarse si la ciencia ha de progresar, tiene que demostrar que sus reglas metodológicas podrían formar la base del sistema

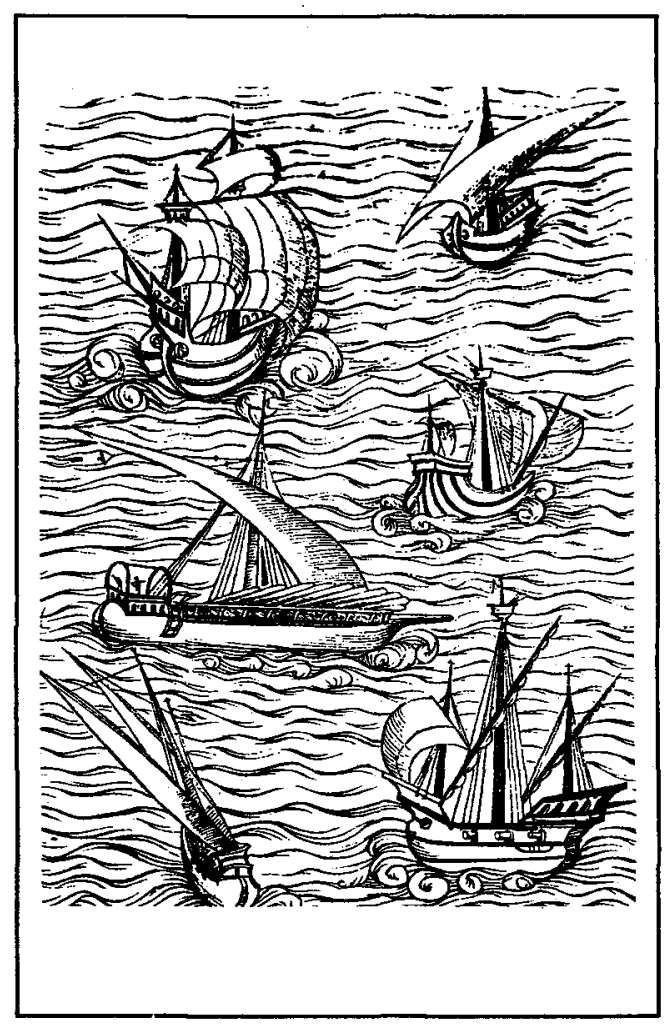

colectivo de toma de decisiones que llamamos ciencia. Entonces, aunque el programa Popperiano no está invalidado por observaciones de que las élites científicas no siguen sus reglas (ver, por ejemplo a Collins, 1975, 1981; Farley y Gerson, 1980; Knorr et al., 1981; Pinch, 1986; Wynne, 1976) porque el programa no trata de describir cómo funciona la ciencia constituida en la actualidad, tiene que mostrar que una comunidad científica podría funcionar de la manera prescrita sino se rechaza como vacua. Localizando la fuente de la validez epistemológica en las convenciones colectivas de las comunidades científicas, Popper tiene que desarrollar una sociología de comunidades científicas posibles como parte principal de una teoría epistemológicamente adecuada del progreso científico. 
Hay por lo menos cuatro razones principales para dudar del éxito del programa Popperiano en la formulación de reglas que conducirían al progreso en el sentido de mejorar el contenido de verdad de las teorías científicas. Primera, falla en especificar en detalle cómo se supone que los científicos decidan cuándo aceptar o rechazar pronunciamientos observacionales básicos donde hay alguna duda sobre los detalles experimentales, $y /$ o la aceptabilidad de teorías observacionales particulares. En tales situaciones, los científicos podrían discrepar bastante "racionalmente" sobre la posible falsificación de las teorías. Sugerir que ajustes ad-hoc sean rechazados es de poca ayuda aquí porque sencillamente no es claro qué posibles modificaciones aumentan o disminuyen el contenido empírico. Así que la adhesión a normas falseadas puede no distinguirse de las estratagemas convencionalistas. Similarmente, Popper asume que el contenido empírico de una teoría es, en principio, medible y comparable a través de teorías y sin embargo no ofrece sugerencias de cómo debe lograrse ésto y por consiguiente, tampoco pautas para demostrar que el progreso intelectual, de hecho, ha ocurrido. Así que, como teoría de cómo progresa la ciencia, es incompleta.

Segunda, comparar y decidir entre dos teorías en competencia, incluye la comparación de sus teorías observacionales asociadas, condiciones de aplicación, vocabulario descriptivo, etc. (Feyerabend, 1968). Parece haber poca razón para esperar que todas ellas traslapen en la extensión requerida para que un solo experimento crucial decida sin ambigüedad entre ellas. Entre más divergentes sean los términos por medio de los cuales se expresan las teorías y los dominios sobre los que pretenden ser verdaderas, es menos probable que puedan compararse y evaluarse directamente. Dadas las condiciones necesarias entre teorías observacionales y teorías generales (i.e. deben compartir términos descriptivos básicos y ser mutuamente consistentes) podría ser bastante "racional" para los adherentes de una teoría, rechazar la evidencia producida por los seguidores de una en competencia. Claramente, las teorías que conciben los fenómenos de maneras radicalmente diferentes, no pueden evaluarse en términos de un solo conjunto de afirmaciones básicas (Lakatos, 1970).

Tercera, no es del todo obvio el porqué la competencia evolucionaria, deba conducir necesariamente al progreso cognitivo, excepto en el sentido trivial de que la supervivencia define la superioridad. Para que la prueba de ensayo y error sea progresiva en un sentido Popperiano, los dominios de teorías en competencia tendrían que ser directamente comparables $\mathrm{y}$ estables en una secuencia de exámenes. Igualmente, el significado de cada examen y sus condiciones de validez tendrían que ser claros y generalmente aceptables para que se puedan determinar conclusiones "progresivas". La competencia entre teorías implica entonces un dominio estable y común de aplicación si ha de ser progresiva. De otra manera, no hay razón para esperar que las teorías sobrevivientes sean "mejores" que otras. Popper no ofrece fundamentos para presumir tal comunidad entre teorías rivales, fuera del punto de vista de que si la ciencia ha de progresar entonces tiene que existir. Como lo puntualiza Feyerabend 
en una serie de trabajos $(1965,1975$, 1981), hay considerable evidencia histórica para sugerir que otras formas de progreso intelectual han ocurrido cuando éste no existía.

Estas y otras dificultades en el programa Popperiano condujeron a Lakatos a desarrollar su bien conocida metodología de programas de investigación científica (1970) que intentaban formular una teoría "racional" del cambio científico mientras permitían supuestos improbables y el rechazo de evidencia conflictiva. Más tarde (1971) extendió este planteamiento para incorporar la historia de la ciencia en un intento para integrar teorías epistemológicas con reconstrucciones históricas. Sin embargo, como lo señala Feyerabend (1970), Lakatos solamente es capaz de reivindicar la racionalidad del progreso científico por medio de la competencia entre programas de investigación en retrospecto, y aún entonces los veredictos de la historia pueden ser revisados a través de la revitalización de programas rechazados. Así la racionalidad se hace contingente y contextual.

Además de estos problemas internos hay el tema más sociológico de si es posible, o aún concebible, que las comunidades científicas puedan organizarse alrededor de la meta común de la búsqueda de la verdad corregible por medio de la falsificación sistemática de las creaciones intelectuales de los miembros por procedimientos de prueba y criterios acordados en común para evaluar la validez de las afirmaciones básicas. No sólo tales organizaciones sociales parecen extremadamente raras en la historia de las ciencias occidentales modernas, sino que es difícil ver cómo podrían establecerse y reproducirse.

Una institución social que premia la producción de conjeturas atrevidas $\mathrm{y}$ teorías radicales y su elaboración en detallados informes de fenómenos y eventos, anima el compromiso con esas teorías $\mathrm{y}$ no con su falsificación $\mathrm{y}$ rechazo. Tal compromiso es posible que conduzca al rechazo de la evidencia contradictoria y el sistema de creencias que lo produce. Si por otra parte, la institución social premia la crítica y la demolición de teorías, es difícil ver por qué los científicos deben molestarse en desarrollarlas, mucho menos en hacerlas fáciles de falsificar. La investigación necesita tiempo y recursos, de modo que si no es apoyada por las instituciones que gobiernan la asignación de recompensas, entonces no es probable que sea emprendida extensivamente. Así, un sistema intelectual enteramente crítico es probable que agote las conjeturas para criticar y por consiguiente es improbable el progreso. Una institución social que trate de premiar ambos conjuntos de actividades es probable que se bifurque en productores de teorías y destructores de teorías que comparten pocos intereses y supuestos comunes. Exactamente cómo la combinación ideal de conjeturas y refutaciones de Popper puede lograrse en una institución social actuante, permanece, en consecuencia, oscura. Esta oscuridad no se reduce mayormente con sus argumentos políticos en favor de sociedades "abiertas". Puesto que es la institucionalización colectiva de sus reglas metodológicas lo que genera el progreso, esta oscuridad significa que su teoría del progreso científico es incompleta. 
Reglas metodológicas en las ciencias humanas y papel de la investigación contable en las cambiantes prácticas.

Estos problemas y dificultades del programa Popperiano en la filosofía de la ciencia producen que su aplicación directa a las ciencias humanas a la manera de Watts y Zimmerman sea de dudoso valor. Aún si fuera más exitoso al dar cuenta del progreso en las ciencias naturales, sin embargo, no es obvio que tales reglas metodológicas se apliquen necesariamente a las ciencias humanas. Hay tres conjuntos de razones principales de por qué la simple extensión de reglas metodológicas para el estudio de los fenómenos naturales en las ciencias humanas es cuestionable. En primer lugar, las metas de las ciencias humanas no son necesariamente idénticas a los propósitos dominantes de las ciencias naturales modernas, i.e. el control sobre el mundo natural y por consiguiente las reglas metodológicas justificadas en términos de este último, pueden no ser apropiadas. En segundo lugar, la investigación social es una actividad interactiva y cargada de valor en formas que no se aplican a las ciencias naturales. En tercer lugar, los fenómenos y eventos sociales están constituidos de significados y convenciones culturales y por ende, varían.

En relación con el primer punto, la mayoría de las filosofías de las ciencias naturales han asumido que su propósito fundamental era mejorar nuestra habilidad para manipular y controlar el mundo natural, en lugar de por ejemplo, admirar y llegar a integrarse con procesos naturales ordenados coherentemente. En este sentido, siguen la ideología dominante de la ciencia occidental moderna desde la última parte del siglo XVII y el triunfo del programa Baconiano para el dominio de la naturaleza (e.g. Daela, 1977; Merchant, 1980, pp. 172-190). Como resultado, la mayoría de las metodologías normativas conciben la validez y el progreso epistemológicos en términos de predicción y control. Mientras que tales motivaciones están indudablemente presentes en las ciencias humanas y ciertamente ellas probablemente constituyen la razón de la mayor parte de la financiación de la investigación social, no son las únicas y no monopolizan todas las tradiciones de la investigación y las pautas de evaluación. En particular, hay convenciones y normas bien establecidas que gobiernan la investigación histórica y los estudios literarios que no están justificados en términos de metas de control y que hasta ahora han resistido exitosamente la subordinación a las doctrinas lógicoempiristas (Stockman, 1983, pp. 128130). Aún si el interés dominante al emprender una investigación sobre contabilidad -e investigación sobre administración en general-es generar conocimiento que ayude a resolver problemas de los profesionales, habilitándolos para controlar mejor los procesos sociales, la existencia de metas alternas y normas metodológicas, quiere decir que la presunción de la unidad de las reglas metodológicas a través de todas las ciencias, no está garantizada. En cualquier caso, la idea de que el conocimiento científico social puede mejorar la habilidad de los gerentes para controlar el mundo social como si fuera un sistema social cerrado, cuyas salidas puedan ser 
variadas sistemáticamente alterando las entradas, es dudoso como lo vamos a ver.

El problema de ser la investigación social de naturaleza cargada de valor, ha sido el tema de extensas discusiones, por lo menos desde los ensayos de Weber sobre sociología (1949), y Schreuder ha suministrado recientemente un resumen útil de algunos de los puntos principales en cuestión, con referencia particular a teorías de la contabilidad (1984). Como él sugiere, tanto las ciencias naturales como las ciencias sociales y las escuelas metodológicas que se ocupan de ellas, incluyen juicios de valor en el diseño de proyectos de investigación, en la aplicación práctica de resultados y en el compromiso general con la investigación como una actividad que conduce al conocimiento. Difieren, sin embargo, en el grado en que: (a) los juicios evaluativos son parte de las descripciones adecuadas de los fenómenos, y (b) los informes científicos abarcan necesariamente evaluaciones críticas de los fenómenos que están siendo estudiados.

Las descripciones de los eventos y fenómenos sociales a menudo requieren el uso de términos que tienen connotaciones positivas o negativas, tanto en la propia cultura del científico como en la que está siendo objeto de análisis.

Democracia, campos de exterminio y eficiencia son todos términos que pueden usarse para proveer descripciones precisas de los estados de negocios y que tienen significados evaluativos. Así, su uso correcto necesariamente implica juicios de valor sobre el estado de los negocios que se describen en una forma que no ocurre en muchas ciencias naturales -aunque pueden hacerlo en las ciencias biológicas. En y por sí misma, esta necesidad no implica que las ciencias sociales son fundamentalmente diferentes de la física y de la química ya que las reglas para evaluar términos y frases descriptivos pueden desarrollarse de manera similar a aquellas en las áreas mencionadas sin adherir necesariamente a juicios de valor implícitos en ellas. Así, hay convenciones que gobiernan la descripción correctade un sistema político como una democracia y como un campo de prisioneros, un campo de exterminio (Bhaskar, 1979, pp. 74-77), que no pueden distinguirse de compromisos de valor implicados en esos términos. Sin embargo, este componente evaluativo de términos descriptivos sí restringe su dominio de aplicación apropiada y por consiguiente la generalidad de afirmaciones con características legales que las incluyen. Aplicar términos como democracia, justicia y libertad al análisis de sociedades que no tienen un concepto de lo que significan o cómo podrían ser valoradas, es claramente producir descripciones inadecuadas. Reglas metodológicas que insistan en la formulación de afirmaciones universales, son, entonces, inaplicables a las ciencias humanas.

La construcción de explicaciones sociales científicas de eventos y de fenómenos implica que las explicaciones y descripciones cotidianas son de alguna manera, defectuosas e inadecuadas. Así, buscar la explicación de por qué ciertas empresas usan convenciones contables particulares para presentar sus resultados en el propio interés de sus juntas directivas, como lo hacen muchos investigadores mencionados por Watts y Zimmerman, es criticar las propias versiones de los 
gerentes en cuanto a sus preferencias, i.e. como necesitando mayor explicación. Tales explicaciones, que necesariamente envuelven redescripciones de los informes de los participantes, también sugieren por qué los gerentes sostienen las creencias incorrectas que dicen tener. Puesto que estas creencias e informes constituyen la realidad social que está siendo explicada, entonces los científicos sociales están criticando inevitablemente lo que existe, sencillamente tomando esas creencias e informes como problemáticos. De este modo, la afirmación de la superioridad epistemológica por parte de los investigadores implica una evaluación negativa de los signifrcados cotidianos. Exceptuando quizás el uso de ciertas metáforas, este tipo de evaluación de los fenómenos estudiados no ocurre en las ciencias naturales, por lo menos no con el mismo alcance (Merchant, 1980, pp. 192-252), y de esta manera, teorías del método científico que lo prohiben no son aplicables a las ciencias humanas.

Estas diferencias entre las ciencias naturales $\mathrm{y}$ las ciencias humanas están conectadas al tercer conjunto de razones para cuestionar la directa aplicabilidad de reglas metodológicas del estudio del mundo natural al mundo social: la naturaleza significativa de los fenómenos sociales. Los objetos que se explican en las ciencias sociales están constituidos en parte por los significados usados para caracterizarlos por parte de la gente y por las normas que gobiernan los usos correctos en culturas particulares. Así, el mismo movimiento físiologico, e.g. el apretón de manos, puede tener significados bastante diferentes en culturas diferentes y por consiguiente constituyen fenómenos sociales diferentes. Los informes financieros pueden consistir de marcas negras en el papel pero sus significados varían de acuerdo con las convenciones que rigen su uso y por consiguiente la naturaleza del informe financiero depende del sistema de significados del cual es una parte. De esta manera, cualquier descripción de un "hecho" social incluye una interpretación de símbolos culturales y de los significados usados en la vida diaria. Esta interpretación de descripciones de otros abarcauna interacción entre los científicos sociales y los fenómenos estudiados de un modo que no ocurre en las ciencias naturales. En el último caso, los científicos pueden crear su propio lenguaje de observación con propósitos teóricos sin tener que considerar si sus descripciones están conectadas a las de los participantes. Los científicos sociales, por otra parte, tienen que comprender los significados cotidianos de símbolos y acciones en contextos culturales particulares si los han de utilizar como objetos de investigación. Descripciones adecuadas de los fenómenos sociales requieren entonces alguna comprensión de las concepciones de los participantes ya que éstas forman la "base empírica". Por ejemplo, cualquier estudio comparativo de la profesión contable en Europa tendría que apreciar que la idea inglesa de "profesión" es diferente de la sostenida por muchas culturas continentales y por consiguiente el significado del término "Contador Profesional" varía entre culturas nacionales (Friedson, 1986, pp. 30-37; Macdonald, 1985). Las normas metodológicas para las ciencias humanas tienen entonces que incluir criterios para evaluar la suficiencia de las descripciones que incorporan la interpretación "correcta" 
de los eventos, en términos de las convenciones culturales dominantes.

La naturaleza significativa de los fenómenos sociales tiene además tres consecuencias mayores para las teorías metodológicas, y también implicaciones para la posibilidad de teorías de los procesos sociales que habilitarían a los contadores y a los gerentes para controlar los resultados más efectivamente a la manera defendida por Watts y Zimmerman.

Primero, las leyes universales son improbables, si no imposibles, puesto que las lenguas, los significados y por ende los fenómenos varían grandemente y están sujetos al cambio. De modo que comparar teorías en términos de su generalidad puede no ser una manera útil de valorar sus méritos. Teorías sumamente generales como la economía neo-clásica pueden ser inferiores a otras más específicas sencillamente porque hay pocos fenómenos, si los hay, a los cuales se refieren.

Segundo, las afirmaciones básicas no son simplemente construidas por los investigadores sino que, como lo acabamos de ver, incluyen descripciones y teorías cotidianas. Si estas últimas cambian, entonces también lo hacen los criterios para valorar la suficiencia de las descripciones científicas y por consiguiente su generalidad. Entonces, lo que podría haber sido una descripción aceptable para "probar" una teoría en ciertas condiciones, se torna inaceptable cuando esas condiciones se alteran. Además, las descripciones de todos los días pueden ser impugnadas por grupos en conflicto al interior de comunidades de lenguas y culturas de tal manera que los estándares para la evidencia evaluativa que presume un consenso de actores sociales puede ser inaplicable. Teorías de método científico que requieren acuerdo científico en lo que cuenta como una afirmación básica, tal como la de Popper, tienen que extender ese requisito a los informes de los participantes -en cuyo caso pueden no ser relevantes para mucha investigación social- o desarrollar reglas para tratar descripciones conflictivas sobre lo que sucedió. Tales reglas, por supuesto, han sido desarrolladas por historiadores y otros científicos humanistas "interpretativos" pero éstas típicamente no encajan en la clase de normas metodológicas preferidas por los adherentes a la doctrina de la unidad de la ciencia y un solo "método científico". Nuevamente, esta doble impugnación y revisión de la evidencia empírica implica la limitada naturaleza de las afirmaciones generales en las ciencias humanas y el limitado dominio sobre el cual las teorías pueden ser válidas.

Tercero, la naturaleza significativa de los fenómenos sociales implica que están interrelacionados conceptualmente y en consecuencia varían cuando cambian sus relaciones con otros fenómenos. Esto implica que las relaciones entre entidades sociales son internas. Dos o más objetos están relacionados internamente cuando el ser un tipo particular de objeto social depende de las conexiones del uno con el otro (Harré, 1979, pp. 88-89). Por ejemplo, para ser esposo, pariente o empleado, una persona tiene que ser casada, tener hijos o recibir un salario de un patrón que tiene ciertos derechos de propiedad sobre el uso de las capacidades de uno. Si estas últimas 
relaciones no se sostienen, entonces las descripciones previas de los roles sociales, son falsas. Así, la naturaleza de los fenómenos sociales particulares depende de cómo están relacionados con otros fenómenos. Esto quiere decir que las explicaciones causales que requieren de la causa y el efecto para ser ontológicamente separadas no son aplicables a los procesos sociales. Así que las teorías que están modeladas en la mecánica Newtoniana y que presumen que las relaciones cambiantes entre los fenómenos no cambian su naturaleza, son inapropiadas para la explicación de los procesos sociales y por supuesto tampoco son apropiadas para áreas significativas de las ciencias naturales (Prigogine y Stengers, 1984).

Por ejemplo, el crecimiento de grandes jerarquías gerenciales en los Estados Unidos se explica en términos de su relativa eficiencia en integrar la producción en masa con las prácticas comerciales masivas en circunstancias particularmente favorables (Chandler, 1977, 1981) y/o en reducir los costos transaccionales en situaciones de mucha incertidumbre (Williamson, 1975; Kay, 1984, pp. 79-100; Daems, 1983). El mecanismo productivo fundamental en estos informes es la competencia imperfecta realizada y mediada por arreglos institucionales particulares. Sin embargo, al generar tales jerarquías, estos arreglos y la operación de los mercados competitivos cambian ellos mismos, como se evidencia por la habilidad de grandes firmas para diversificar y cambiar las fronteras del mercado (Karpik, 1978). Así los mecanismos causales pueden producir fenómenos particulares en situaciones particulares, pero al hacerlo, se

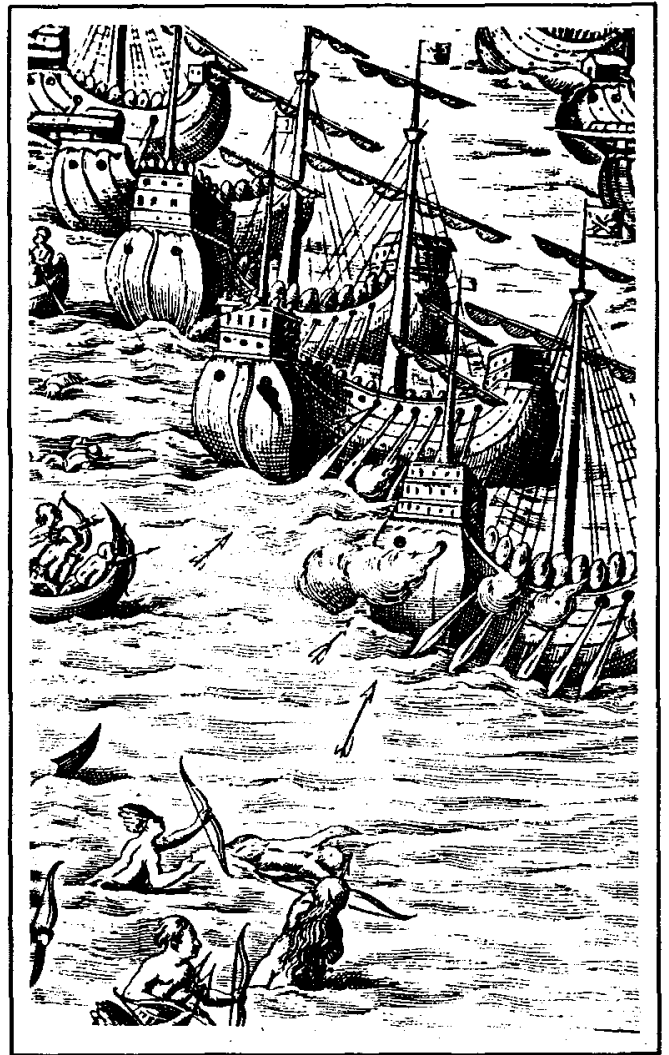

alteran ellos mismos a causa de las relaciones internas entre las entidades sociales. Las metodologías que requieren leyes causales universales que expresen relaciones externas, estrictamente determinísticas entre las estructuras y los efectos, son, entonces, inapropiadas para las ciencias sociales.

Estas diferencias entre las ciencias sociales y naturales significan que las normas metodológicas para evaluar pretensiones de conocimiento en las primeras no son de ningún modo aplicables a las últimas. Además están conectadas a una característica diferenciadora adicional: los fenómenos sociales sólo ocurren en sistemas "abiertos" mientras que los fenómenos naturales pueden ser generados en sistemas cerrados por experimen- 
tos de laboratorio (Bhaskar, 1979, pp. 57-62). Los sistemas abiertos son aquellos en los que las regularidades invariantes empíricas no obtienen y por consiguiente no pueden dar pruebas de teorías a la manera preconizada por muchas metodologías de las ciencias naturales. Puesto que es sólo en sistemas cerrados que las conjunciones constantes entre los eventos pueden producirse, las predicciones de los eventos sociales son siempre tendenciales y nunca determinísticas.

Junto con la conexión interna de los fenómenos sociales, este punto implica severas restricciones en el desarrollo de teorías formales de los procesos sociales que podrían habilitar a gerentes, inversionistas o cualquier otro para lograr resultados específicos variando sistemáticamente los insumos particulares. Parece irrealizable la idea de que la investigación científica conduce a la construcción de teorías cuantitativas precisas y completas de fenómenos sociales que podrían ser incorporadas a técnicas contables y administrativas, en forma similar a las teorías de máquinas ideales y descargas de electrones en bombillas eléctricas que están siendo incorporadas a los métodos de diseño de la ingeniería (Channell, 1982; Reich, 1985, pp. 121126). La pretensión de Watts y Zimmerman de que la investigación contable habilita a los profesionales para tomar mejores decisiones, porque eso les dice cuáles son las consecuencias de las diferentes alternativas, implica que el sistema en manipulación está cerrado, bien comprendido e independiente de las creencias y acciones de los profesionales. Una vez que se está de acuerdo en que estas condiciones no se sostienen, entonces la investigación contable tiene que ser justificada en términos de otros. criterios y otros fines.

En lugar se buscar alguna teoría idealizada de sistemas sociales cerrados al estilo de la economía neo-clásica como parecen desearlo Watts y Zimmerman y otros discípulos de Chicago, estos puntos sugieren que los investigadores de la contabilidad interesados en comprender las prácticas contables con el punto de vista de "mejorarlas" de alguna manera deberían volverse más modestas en sus metas y centrarse en cómo los procesos sociales particulares ayudaron a producir conjuntos de prácticas particulares en ciertas circunstancias. La constitución y cambio de las prácticas contables como maneras de generar varios tipos de información para diferentes grupos, se convierten entonces en objetos que requieren explicación, en lugar de "realidades" dadas por supuestas. Tales explicaciones necesariamente abarcan temas epistemológicos ya que la información contable pretende describir realidades sociales para usuarios que pueden actuar con base en ella (Mattessich, 1972). Cualquier teoría de prácticas contables que las considere problemáticas, cuestiona su suficiencia como convenciones productoras de conocimiento y reclama un estatus epistemológico superior. $\mathrm{Al}$ hacerlo, implica prácticas contables alternas que serían mejores porque se basan en reglas metodológicas que presumen generar conocimiento superior para los informes cotidianos. Puesto que las prácticas contables generan información y conocimiento, entonces una teoría socialde cómo y por qué se desarrollaron esás prácticas y operan como lo hacen, presupone la superioridad de su marco de referencia para producir conocimiento 
que las explique, y, presumiblemente este marco de referencia puede ampliarse para mejorar su validez.

Por ejemplo, si se pretende que un cambio particular en las convenciones contables se debe "realmente" a las presiones de poderosas élites comerciales sobre los contadores, en lugar de los avances técnicos basados en la teoría contable normativa, ésto implica que el científico social o el investigador contable tiene un conjunto de reglas metodológicas que la o lo habilitan para producir conocimientos más válidos que aquel en el que confían FASB o entidades similares. Este conocimiento "mejor" reconstruye el cambio y las racionalizaciones aducidas en su favor como fenómenos que deben ser explicados por una teoría social de prácticas contables más amplia y más completa. Como tal, esta teoría, en principio, es usada por entidades contables estándar para formular mejores políticas que en el pasado, relativas a propósitos específicos, y así aumentar el conocimiento sobre sus propias prácticas y sobre la validez de sus justificaciones, $y$, al mismo tiempo, dar algunas pautas sobre la producción de información más válida acerca de las prácticas sociales.

Entonces, desde este punto de vista, la investigación contable debería dar no solamente explicaciones de por qué ocurren y cambian las prácticas particulares, sino por qué los profesionales han llegado a tener sobre ellas creencias falsas o incompletas. Es decir, las explicaciones científicas sociales, son superiores a los informes diarios de los eventos en tanto que dan razones acerca de las creencias equivocadas sobre even- tos y fenómenos así como mejores informes de ellos. Tales explicaciones podrían mejorar las prácticas contables si los profesionales modificaran sus creencias y acciones en consecuencia y por consiguiente la "aplicación" de las teorías sociales se asemeja a un proceso de "ilustración" en lugar de insumos modificados para un sistema relativamente cerrado. $\mathrm{Al}$ proporcionar mejor comprensión de por qué surgen convenciones particulares y cómo funcionan, los investigadores contables deberían habilitar a los profesionales para mejorar las prácticas y procedimientos que producen información contable para propósitos específicos en situaciones específicas. Por supuesto, este proceso cambiaría las realidades sociales de tal manera que hace inaplicable la explicación científica social original.

\section{Surgimiento de la investigación contable moderna}

Si el tipo de investigación resumido y defendido por Watts y Zimmerman no sigue las reglas metodológicas a las que ellos pretenden adherir, $y$, en muchos casos éstas ni son características de las ciencias naturales, ni libres de la incoherencia interna ni aplicables directamente a las ciencias sociales, entonces uno se preguntaría por qué se ha hecho tanta investigación de este tipo en los Estados Unidos y en otras partes en las últimas dos décadas. Aunque una parte importante de la respuesta a esta pregunta es la prevalente jerarquía de prestigio académico y la facilidad relativa para realizar y publicar investigación cuantitativa utilizando datos que puedan ser leídos por las máquinas y públicamente 
disponibles, comparados a los estudios de campo (Hopwood, 1983; Kaplan, 1986), ésto no explica por qué tal jerarquía de gran prestigio se institucionalizó en los Estados Unidos en los 60, ni por qué las élites profesionales aparentemente aceptan su dominio en los programas de entrenamiento universitario.

Una completa discusión de estas cuestiones no puede darse aquí, pero pueden indicarse algunos de los factores $\mathrm{e}$ influencias mayores. Esencialmente, el desarrollo de la investigación contable moderna puede comprenderse mejor como parte de la expansión general de la educación superior en muchos países industrializados en los 60 y los 70 , combinada con la extendida creencia de que la investigación científica podría no sólo incrementar nuestro dominio sobre el mundo natural sino que podría también resolver los problemas sociales y ayudar a manejar el cambio social. Esta creencia en el conocimiento científico como una fuente crucial en el mantenimiento y mejoramiento del orden social ha sido particularmente fuerte en los Estados Unidos desde la era progresiva (Bledstein, 1976,pp. 123-127,324-331; Wiebe, 1967, capítulo 6) y recibió un considerable refuerzo de su evidente utilidad militar en la segunda guerra mundial (Kevles, 1977, pp. 367-392). Como es bien sabido, el éxito de la investigación de operaciones en esa guerra animó a muchos a creer que técnicas y planteamientos similares podrían producir conocimiento útil para administrar organizaciones sociales y tratar con los problemas sociales (Hall, 1983). Adicionalmente, el largo períodode crecimiento económico en muchas sociedades en los 50 y los
60 , habilitaron a algunos economistas para proclamar exitosamente la validez y utilidad de su conocimiento -hasta el punto que gran número de economistas fueron reclutados por muchas burocracias nacionales (Coats, 1981).

El prestigio y la notoria utilidad de las ciencias naturales, la matemática y la economía animaron a Escuelas Líderes de negocios en los Estados Unidos buscando mejorar su prestigio general, a invertir en la investigación "científica". Esta tendencia fue apoyada directamente por las fundaciones Carnegie y Ford que publicaron informes fundamentales sobre la educación comercial, en 1959 por Gordon \& Howell, y Pierson et al., que pedían una expansión de la investigación "científica" en las escuelas de negocios de los Estados Unidos. Este tipo de investigación hace énfasis en la prueba de hipótesis, en los métodos cuantitativos y en el desarrollo de modelos formales que prometían proveer técnicas para resolver problemas administrativos $\mathrm{y}$ controlar los procesos sociales (e.g. Pierson et al., 1959, pp. 313-314). Estas bases también dieron más apoyo material para esta clase de trabajo en forma de aportes substanciales y oportunidades de publicación que ayudaron considerablemente al desarrollo de más investigación "rigurosa" en Finanzas y Contabilidad (Dyckman y Zeff, 1984).

La popularidad de este tipo de investigación, y su rápida difusión a la mayoría de los campos embrionarios de la investigación administrativa, puede entonces explicarse en parte por su prestigio social contemporáneo y el reclutamiento de matemáticos aplicados, "científicos conductistas" y economistas. Sin em- 
bargo, también dio un número de beneficios a los que buscaban respetabilidad y autonomía académicas al mismo tiempo que presumían de mejorar las técnicas administrativas. Primero, insistiendo en la importancia de los modos de expresión y razonamiento matemáticos, las academias escolares de negocios excluirían a los profesionales del avalúo de su competencia y técnicas de investigación.

Junto con el compromiso para con las teorías económicas abstractas y sumamente formalizadas, ésto aumentó las barreras para entrar y sentó las bases para un sistema de entrenamiento relativamente estandarizado y medios de certificar competencia académica en forma similar a la matematización de cursos universitarios de ingeniería en los años de 1880 y 1890 en los Estados Unidos (Calvert, 1967, pp. 60-80; Levy, 1980, p. 201). El mercado en aumento de trabajo para habilidades investigativas en comercio y administración fue así organizado y controlado por medio de competencias fácilmente reconocidas y comprobadas que podrían ser inculcadas a través de programas formales de entrenamiento.

Segundo, ésto facilitó la producción de gran número de resultados investigativos específicos y altamente restringidos en un amplio rango de tópicos. Las técnicas básicas del modelo matemático y el análisis estadístico puede aplicarse a todo tipo de problemas y temas sin tener que aprender grandes cantidades de detalles descriptivos. En un sistema competitivo en el que los investigadores tienen que publicar rápida y frecuentemente para ganar atención, tenencia y promoción, este estilo de investigación es preferible al que insiste en detalladas y extensas observaciones de prácticas contables en organizaciones, tal como el defendido por Kaplan (1986). También reduce la incertidumbre sobre la validez de los resultados investigativos porque se vuelve una cuestión técnica en lugar de una conceptual o teórica. La competencia entre investigadores en esta situación puede mitigarse fácilmente por la especialización en tópicos diferentes ya que no hay necesidad de demostrar la relevancia teórica de los resultados.

Tercero, basando su estilo investigativo en el modelo mecanicista de sistemas sociales y proclamando la habilidad de ofrecer completa comprensión de tales "máquinas", los investigadores putativos de la contabilidad fueron capaces de combinar barreras de entrada con la promesa de mejores técnicas de control. La búsqueda de afirmaciones generales sobre las relaciones invariantes, que podrían ser trasladadas a los procedimientos administrativos para el manejo de procesos sociales para lograr resultados determinados, justificaron formulaciones abstractas y matemáticas. Se estimuló la medida y la conclusión porque parecían ser "científicas" y daban firmes predicciones sobre los resultados de las decisiones de asignación de recursos. Esta búsqueda de precisión cuantitativa y simplicidad recuerda los intentos de la Oficina de Caminos Públicos de los Estados Unidos por convertir los diseños viales en una actividad científica por medio de pruebas extensivas de laboratorio de las relaciones entre dos o tres variables bajo condiciones artificiales y sumamente restringidas. Comoloaclara Seely (1984), 
no sólo gastaron más tiempo y esfuerzo de lo que se pensó originalmente sino que no provenían de ningún modelo teórico fundamental y resultaron inútiles para los ingenieros practicantes. Las pruebas de campo, ejecutadas por ingenieros estatales que trataron de imitar características críticas de condiciones ambientales y usando patrones, resultaron ser más prácticas e incrementaron significativamente el diseño de carreteras.

Estas ventajas de los métodos cuantitativos y los modelos mecanicistas ayudan a explicar su rápida adopción por investigadores académicos de comercio y administración, ansiosos de demostrar sus poderes científicos. Sin embargo, ellos no explican por qué los profesionales, especialmente aquellos cuyas habilidades podrían considerarse amenazadas por la nueva base "científica" de técnicas prácticas, aparentemente consienten su exclusión de la producción y evaluación del conocimiento. Se podría pensar que probablemente los contadores en particular se oponen a tal "cientificación" de destrezas y prácticas, especialmente en los Estados Unidos y en el Reino Unido donde están organizados como una profesión legítima y con su propia política.

En el caso de los Estados Unidos, esta aceptación del nuevo estilo de investigación contable puede explicarse parcialmente por el prolongado y sostenido dominio del entrenamiento vocacional por parte de facultades y universidades, especialmente desde que las principales facultades privadas establecieron escuelas profesionales a finales del siglo XIX. Similarmente, desde los años 20 , se ha intentado usar la "ciencia" como un medio de legitimar la autoridad experta y de excluir a los advenedizos, como los inmigrantes, del acceso a los cargos elitistas (Auerbach, 1971; Freidson, 1970; Larson, 1977). Así que el basar las habilidades profesionales en el conocimiento académico es una práctica relativamente establecida en los Estados Unidos. Sin embargo, tal conocimiento no necesita ser nomológico y deductivo para realizar estas funciones, como se demuestra con el desarrollo de la "ciencia" legal. Esta consistía de inducción analítica de casos, según Auerbach (1971, p. 552), y formaba la base de una clara ocupación para profesores de derecho y para reformadores.

Sugiero que la aceptación de formas de investigación y conocimiento de la contabilidad, más formales, cuantitativas y deductivas en los Estados Unidos, puede verse como el resultado de tres factores fundamentales. Primero, hasta donde las prácticas y convenciones contables han sido tradicionalmente legitimadas por cualquier posición teórica, han dependido de la economía marginalista (Tinker, 1985, pp. 107-113). Consecuentemente, la integración de la investigación contable con la teoría económica y la adopción del razonamiento económico en la formulación de problemas de investigación, son difíciles de contradecir sin parecer negando la existencia de cualquier base teórica coherente para tal investigación.

Segundo, aunque Watts y Zimmerman y sus colegas reclaman la utilidad de su investigación para los profesionales de la contabilidad, sus "leyes" e hipótesis tienen poca o ninguna implicación en las 
prácticas contables porque no están relacionadas con ellas en ningún sentido significativo. Más bien, la mayor parte de estos estudios tratan de mundos imaginarios de equilibrios económicos donde la información es verdadera y gratis y todo el mundo actúa "racionalmente" y por consiguiente no puede formar la base para reformar y racionalizar las prácticas y habilidades contables en inciertos mundos sociales donde la gente tiene expectativas, metas y creencias heterogéneas. Las prácticas y convenciones actuales no están entonces amenazadas por este tipo de investigación porque no trata con ellas.

Finalmente, la inflación de los 70 y el crecimiento de las intervenciones legales en el sistema financiero hizo las prácticas contables tradicionales, sospechosas y en necesidad de alguna revisión y legitimación adicional. Precisamente como resultado de la inflación de la República de Weimar se incrementó la atención dada a las teorías académicas de entidades contables (Locke, 1984, pp. 163$165,272-273$ ), así mismo estas exigencias en los 70 animaron a la élite profesional para contratar académicos $y$ reclamar implícitamente la autoridad de la "ciencia" para las revisiones propuestas. Parece probable que el crecimiento de la litigación en asuntos contables estimule más esta búsqueda de la justificación "científica" de convenciones y prácticas.

\section{Conclusiones}

La expansión y redirección de la investigación contable en los 60 y los 70 formaron parte de un crecimiento general en la investigación académica de negocios y administración en los Estados Unidos y su orientación hacia normas y procedimientos "científicos". Como tal, ésto reflejó creencias extendidas acerca de la utilidad del conocimiento científico para el manejo de problemas y procesos y la necesidad de reorganizar los programas de entrenamiento alrededor de tal conocimiento. El resultado ha sido la institucionalización de un claro sistema de producción de conocimientos en los Estados Unidos y en otras partes que está separado de los profesionales y estrechamente ligado a la investigación en finanzas. Este campo está dominado por convenciones y valores intelectuales derivados de la economía ortodoxa y, programáticamente por lo menos, de la popularizada filosofía lógico-empirista de las ciencias naturales. La Teoría Positiva de la Contabilidad de Watts y Zimmerman es un intento de confirmar este dominio y de colonizar programas doctorales en contabilidad, con estos valores. Dada la forma en que opera el sistema de carreras académicas en los Estados Unidos, es probable que tengan éxito aunque su análisis es seriamente deficiente y depende de teorías del método científico que son incoherentes e inaplicables a la investigación contable.

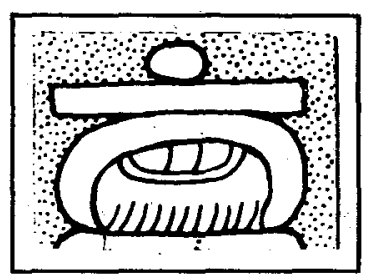




\section{Bibliografias}

\section{El papel de la Teoría Contable}

AMERICAN INSTITUTE OF CERTIFIED PUBLIC ACCOUNTANTS, Objectives of Financial Statements (Trueblood Report). Report of the Study Group on the Objectives of Financial Statements. New York: AICPA, 1973.

ARROW, K.J., Social Choice and Individual Values, Cowles Foundation Monograph. New York: John Wiley, 1963.

R.J. And P. Brown, “An Empirical Evaluation of Accounting Income Numbers”, Journal of Accounting Research 6 (Autumn 1968), pp. 159-178.

BEAVER, W.H., "Financial Ratios as Predictors of Failure", Empirical Research in Accounting: Selected Studies 1966, supplement to Vol, 4 of Journal of Accounting Research (1966), pp. 71-111.

1981.

BENSTON, G.J., Published Corporate Accounting Data and Stock Prices", Empirical Research in Accounting: Selected Studies 1967, supplement to Vol. 5 of Journal of Accounting Research (1967), pp. 1-14 and 22-54.

BLAUG, M., The Methodology of Economics. Cambridge University Press, 1980.

CHAMBERS, R.J., Accounting, Evaluation and Economic Behavior. Englewood Cliffs, N.J.: Prentice-Hall, 1966.

DYCKMAN, T.R., And S.A. Seff, "Two Decades of the Journal of Accounting Research: Journal of Accounting Research 22 (Spring 1984), pp. 225-297.

EDWARDS, E.O., And P.W. Bell, The theory and Measurement of Business Income. Berkeley: University of California Press, 1961.

FRIEDMAN, M., "The Methodology of Positive Economics", Essays in Positive Economics. Chicago: University of Chicago Press, 1953, reimpreso por Chicago: Phoenix Books, 1966.

PASSMORE, J.A., "Can the Social Sciences Be Value-Free?" in H. Feigl and M. Brodbeck, eds., Readings in the Philosophy of Science, pp. 674-676. New York: Appleton-Century-Crofts, 1953, pp. 674-676.

POPPER, K.R., Conjectures and Refutations: The Growth of Scientific Knowledge. London: Routledge \& Kegan Paul, 1963.

New York: Harper Torch Books, 1965. 


\section{Posibilidad y utilidad de la Teoría Positiva de la Contabilidad}

Auerbach, J.S., Enmity and Amity: Law Teachers and Practitioners, 1901)-1922, Perspectives in American History (1971) pp. 551-601.

Bhaskar, R., A Realist Theory of Science (Leeds: Leeds Books, 1975).

Bhaskar, R., The Possibility of Naturalism (Brighton: Harvester, 1979).

Blaug, M., The Methodology of Economics (Cambridge: Cambridge University Press, 1980).

Bledstein, B.J., The Culture of Professionalism (New York: Norton, 1976).

Caldwell, B., Beyond Positivism: Economic Methodology in the Twentieth Century (London: Allen and Unwin, 1982).

Calvert, M.A., The Mechanical Engineer in America, 1830-1900 (Baltimore: John Hopkins University Press, 1967).

Chandler, A.D., The Visible Hand (Cambridge, MA: Harvard University Press, 1977).

Chandler, A.D., Historical Determinants of Management Hierarchies en Van de Ven, A.H. and Joyce, W.F. (eds.) Perspectives on Organization Design and Behaviour, (New York: John Wiley, 1981).

Channell, D.F., The Harmony of Theory and Practice: The Engineering Science of W.J.M. Rankine, Technology and Culture (1982), pp. 39-52.

Christenson, C., The Methodology of Positive Accounting, The Accounting Review (1983) pp. 1-22.

Coats, A.W., Introduction, in Coats A.W. (ed.) Economists in Government (Durham, NC: Duke University Press, 1981).

Coddington, A., Positive Economics, Canadian Journal of Economics (1972) pp. 1-15.

Collins, H., The Seven Sexes: A Study in the Sociology of a Phenomenon or the Replication of Experiments in Physics, Sociology (1975) pp. 205-224.

Collins, H. (ed,), Knowledge and Controversy: Studies of Modern Natural Science, special issue of Social Studies of Science (1981), pp. 1-158.

Daele, W. v.d., The Social Construction of Science en Mendelsohn, E, et al, (eds.) The Social Production of Scientific Knowledge, Sociology of the Sciences Yearbook 1, (Dordrecht: Reidel, 1977).

Daems, H., The Determinants of the Hierarchical Organization of Industry, en Francis A, et al. (eds.) Power, Efficiency and Institutions (London: Heinemann, 1983).

Deane, P., The Scope and Method of Economic Science, Economic Journal'(1983) pp. 1-12.

Dyckman, T.R. \& Zeff, S.A., Two Decades of the Journal of Accounting Research, Journal of Accounting Research (1984) pp. 225-297.

Eichner, A.S., Why Economics is Not Yet a Science, en Eichner, A.S. (ed.) Why Economics is Not Yet a Science (London: Macmillan, 1983).

Farley, J. \& Geison G.L., Science Politics and Spontaneous Generation in Nineteenth Century France: The Pasteur-Pouchet Debate, en Chant, C. and Fauvel, J. (eds.) Darwin to Einstein: Historical Studies on Science and Belief (London: Longman, 1980). 
Fay, Brian, Social Theory and Political Practice (London: Allen and Unwin, 1975).

Feyerabend, Paul K., Problems of Empiricism, en Colodny R. (ed.) Beyond the Edge of Certainty, (Englewood Cliffs, NJ: Prentice-Hall, 1965).

Feyerabend, P.K., How to be a Good Empiricist, en Nidditch, P. (ed.) The Philosophy of Science, (Oxford: Oxford University Press, 1968).

Feyerabend, P.K., Consolidations for the Specialist, en Lakatos, I. and Musgrave, A. (eds.) Criticism and the Growth of Knowledge (Cambridge: Cambridge University Press, 1970).

Feyerabend, P.K. (ed.), Against Method (London: New Left Books, 1975).

Feyerabend, P.K., The Methodology of Scientific Research Programmes, en Feyerabend, P.K. (ed.) Problems of Empiricism, Philosophical Papers, Vol. 2 (Cambridge: Cambridge University Press, 1981).

Findlay, M.C. \& Williams, E.E., A Positivist Evaluation of the new Finance, Financial Management (1980) pp. 7-17.

Findlay, M.C. \& Williams, E.E., A Post-Keynesian View of Modern Financial Economics: In Search of Alternative Paradigms, Journal of Business Finance and Accounting (1985) pp. 1-18.

Freidson, E., Profession of Medicine (New York: Dodd, Mead, 1970).

Freidson, E., Professional Powers (University of Chicago Press, 1986).

Gordon, R.A. and Howell, J.E., Higher Education for Business (New York: Columbia University Press, 1959).

Hall, J.R., An Issue-oriented History of T.I.M.S., Interfaces (1983) pp. 9-19.

Harré, R., The Principles of Scientific Thinking (London: Macmillan, 1970).

Harré, R., Social Being (Oxford: Blackwells, 1979).

Hopwood, A., On Trying to Study Accounting in the Contexts in Which it Operates, Accounting Organizations and Society (1983) pp. 287-305.

Hopwood, A. \& Bromwich, M., Accounting Research in the United Kingdom, en Hopwood, A. and Schreuder, H. (eds.) European Contributions to Accounting Research (Amsterdam: Free University Press, 1984).

Hutchison, T., On the History and Philosophy of Science and Economics, en Latsis, S. (ed.) Method and Appraisal in Economics (Cambridge University Press, 1976).

Hutchison, T., Our Methodological Crisis, en Wiles, P. and Routh, G. (eds.) Economics in Disarray (Oxford: Blackwells, 1984).

Jensen, M.C., Capital Markets: Theory and Evidence, Bell Journal of Economics and Management (1972) pp. 357-398.

Kaplan, R.S., The Role for Empirical Research in Management Accounting, Accounting, Organizations and Society (1986) pp. 429-452.

Karpik, L., Organizations, Institutions and History, en Karpik, L. (ed.) Organization and Environment (London: Sage, 1978).

Kay, N.. The Emergent Firm (London: Macmillan, 1984). 
Kevles, D., The Physicist (New York: Alfred A. Knopf, 1977).

Knorr, K., Krohn, R. and Whitley, R. (eds.), The Social Process of Scientific Investigation Sociology of the Sciences Yearbook 4 (Dordrecht: Reidel, 1981).

Lakatos, I., Falsification and the Methodology of Scientific Research Programmes, en Lakatos, I. and Musgrave, A. (eds.) Criticism and the Growth of Knowledge (Cambridge University Press, 1970).

Lakatos, I., History of Science and its Rational Reconstructions, en Buck, R. and Cohen, R. (eds.) Boston Studies in the Philosophy of Science 8 (Dordrecht: Reidel, 1971).

Larson, M.S., The Rise of Professionalism (University of California Press, 1977).

Levy, R.M., The Professionalization of American Architects and Civil Engineers, Tesis de PhD no publicada, University of California at Berkeley (1980).

Locke, R.R., The End of the Practical Man: Entrepreneurship and Higher Education in Germany, France and Great Britain, 1880-1940 (Greenwich, CT: JAI Press, 1984).

Lowe, E.A., Puxty, A.G. \& Laughlin, R.C., Simple Theories for Complex Processes: Accounting Policy and the Market for Myopia, Journal of Accounting and Public Policy (1983) pp. 19-42.

Macdonald, K.M., Social Closure and Occupational Registration, Sociology (1985) pp. 541-556.

Mattessich, R., Methodological Preconditions and Problems of a General Theory of Accounting, Accounting Review (1972) pp. 469-487.

Merchant, C., The Death of Nature (New York: Harper and Row, 1980).

Musgrave, A., Unreal Assumptions in Economic Theory: The F-Twist Untwisted, Kyklos (1981), pp. 377-387.

Peasnell, K.V. \& Williams, D.J., Ersatz Academics and Scholar-Saints: the Supply of Financial Accounting Research, Abacus (1986) pp. 121-135.

Pierson, F.C., et al., The Education of American Businessmen (New York: McGraw-Hill, 1959).

Popper, K., Conjectures and Refutations (London: Routledge and Kegan Paul, 1963).

Popper, Karl, The Logic of Scientific Discovery (London: Hutchinson, 1968).

Pinch, Trevor, Confronting Nature (Dordrecht: Reidel, 1986).

Prigogine, I. \& Stengers, I., Order out of Chaos (London: Heinemann, 1984).

Reich, L.S., The Making of American Industrial Research (Cambridge University Press, 1985).

Ross, S., The Current Status of the Capital Asset Pricing Model, Journal of Finance (1978) pp. 885-901.

Ryan, R.J., Capital Market Theory - A Case Study in Methodological Conflict, Journal of Business Finance and Accounting (1982) pp. 443-458.

Schreuder, H., Positively Normative (Accounting) Theories, en Hopwood, A. and Schreuder, H. (eds.) European Contributions to Accounting Research (Amsterdam: Free University Press, 1984).

Seely, B.E., The Scientific Mystique in Engineering, Highway research at the Bureau of Public Roads 1918-1940, Technology and Culture (1984) pp. 798-831.

Stockman, N., Antipositivist Theories of the Sciences (Dordrecht: Reidel, 1983). 
Tinker, T., Paper Prophets (New York: Praeger, 1985).

Thomas, D., Naturalism and Social Science (Cambridge University Press, 1979).

Thomas, T.T., Towards a Value-neutral Positive Science of Accounting, Journal of Business Finance and Accounting (1981) pp. 549-572.

Tomkins, C. \& Groves, R., The Everyday Accountant and Researching his Reality, Accounting, Organizations and Society (1983) pp. 361-374.

Turner, S.P., Sociological Explanation as Translation (Cambridge University Press, 1980).

Watts, R.L. \& Zimmerman, J.L., Positive Accounting Theory (Englewood Cliffs, NJ: Prentice-Hall, 1986).

Whitley, R., The Fragmented State of Management Studies, Reasons and Consequences, Journal of Management Studies (1984) pp. 331-348.

Whitley, R., The Transformation of Business Finance into Financial Economics: The Roles of Academic Expansion and Changes in U.S. Capital Markets, Accounting Organizations and Society (1986) pp. 171-192.

Weber, M., The Methodology of the Social Sciences (New York: Free Press, 1949).

Wiebe, R.H., The Search for Order, 1877-1920 (New York: Hill and Wang, 1967).

Williamson, Oliver E., Markets and Hierarchies (New York: Free Press, 1975).

Wynne, Brian, C.G., Barkla and the J. Phenomenon, Social Studies of Science (1976) pp. 307-347.

\section{Políticas y normas contables}

\section{en una muestra de sociedades anónimas del Valle del Cauca}

ACUÑA M. Henao H. Prácticas Contables en el Valle del Cauca. Revista Cuadernos de Administración, No. 11 Univalle 1985, pp. 39-53.

BARONA B., Burbano J. y Sinisterra G. Programa de Investigación en Información Financiera Pública, Mimeo. Univalle 1987.

BALLESTEROS Enrique. Teoría y Estructura de la Nueva Contabilidad. Alianza Universidad Textos. Madrid 1979.

BALL Ray y FOSTER George. Corporate Financial Reporting. A Methodological Review of Empirical Research. Vol 20. USA 1982.

BELKAOUI Ahmed. Accounting Theory Editorial H.B.J. Inc. New York 1981.

BEAVER William. Financial Reporting and Accounting Revolution. Editorial PHI. New Jersey 1981.

BLANCO L. Yanel. Las normas de Contabilidad en Colombia. Edit. Roesga 1987.

BRIONES Guillermo. La Formulación de Problemas de Investigación Social. Ediciones Uniandes. Bogotá 1981.

BRUNS y De Coster D. La Contabilidad y el Comportamiento Humano. Editorial Trillas: México 1975. 
CARDONA John. Estructura Básica de la Contabilidad para Colombia. Revista No. 14 Contaduría Universidad de Antioquia. Medellín 1989, pp. 43-111.

CARDONA John. La Revelación Contable - Un Estudio Exploratorio. Revista No. 8 Universidad de Antioquia. Medellín 1986, pp 77-111.

Cámara de Comencio de Cali. Boletín Estadístico sobre Inversión Privada en el Valle del Cauca. Cali 1986.

Cámara de Comercio de Bogotá. Decreto 2160 de 1986.

CAÑIBANO Leandro. Teoría Actual de la Contabilidad. Biblioteca de Ciencias Empresariales Ediciones ICE. Madrid 1979.

GEORGE Claude. Historia del Pensamiento Administrativo Editorial PHI. México 1984.

HANAN Mack. Incremento de utilidades. Cómo acelerarlo mediante la aplicación de estrategias empresariales. Editorial Norma. Bogotá 1982.

HENDRICKSEN Eldon. Teoría de la Contabilidad. Edit. Uteha. México 1981.

HORNGREN Charles. Contabilidad Administrativa Introducción. Edit PHI. Carvajal. Cali 1983.

KAST. F. y ROSENZWEIG J. Administración en las Organizaciones. Un enfoque de Sistemas. Edit. Mc Graw Hill. México 1980.

MATTESICH Richard. Un examen científico aplicado para una estructura metodológica. Revista Teuken No. 3. C. Rivadavia. Argentina 1988.

MCNICHOLS Thomas. Política Empresarial. Edit. Mc Graw Hill. Bogotá 1981.

MLLER Martin. Guía de Principios de Contabilidad generalmente aceptados. Editorial H.B.J. New York 1982.

MORRISSEY Leonard. Teoría Contable de la Información Financiera. Editorial Trillas. México 1977.

LEE Thomas. Developments in Company Financial Reporting a History and an Introduction. Philip Alan Publishers Limited. Oxford 1981.

LIBBY Robert. Accounting and Human Information Processing: Theory and Aplications. Edit. Prentice Hall Inc. Englewood Cliffs. New Jersey 1981.

PARDINAS Felipe. Metodología y Técnicas de Investigación en Ciencias Sociales. Editorial "Siglo XXI Editores". México 1983.

RODRIGUEZ Mario. La información contable y su exposición. Administración de Empresas. Tomo II.

SHIM J. y SIEGEL J. Contabilidad Administrativa - Ser ie Schaum. Editorial Mc Graw Hill. Bogotá 1986.

SIMKIN M. y MOSCOVE Stephen. Accounting Information Systems Concepts and practice for effective decision. Makin Edit. John Wiley y Sons Inc. New York 1979.

TUA Pereda Jorge. Principios y Normas de Contabilidad, impreso en la Fábrica Nacional de Moneda y Timbre. Madrid 1983.

UDUAL. IV Conferencia de Facultades y Escuelas de Contaduría Pública de América Latina - III Congreso Latinoamericano de Investigación Contable. Universidad Central. Bogotá 1986. 
VASQUEZ José. Contabilidad Moderna. Edit. Bedout. Medellín 1947.

VLAEMMINCK Joseph. Historia y doctrinas contables. Editorial Index. Madrid 1961.

WATTZ y ZIMMERMAN. Positive Accounting Theory. Editorial PHI. New Jersey 1986.

ZEFF Stephen. Evolución de la Teoría Contable. La investigación empírica. Revista Contaduría Universidad de Antioquia. No. 6 Marzo de 1985, pp 25-53. 